\title{
Bound states in the continuum in the visible emerging from out-of-plane magnetic dipoles: Supporting Information
}

Shunsuke Murai, ${ }^{*,+\ddagger}$ Diego R. Abujetas, $₫$ Gabriel W. Castellanos,,$^{\ddagger}$ José A. Sánchez-Gil, ${ }^{*}$, Feifei Zhang, ${ }^{\dagger}$ and Jaime Gómez Rivas ${ }^{*, \ddagger}$

$\dagger$ †epartment of Material Chemistry, Graduate School of Engineering, Kyoto University, Nishikyo-ku, Kyoto 615-8510, Japan

$\ddagger$ Department of Applied Physics and Institute for Photonic Integration, Eindhoven

University of Technology, P.O. Box 513, 5600 MB Eindhoven, The Netherlands

9Instituto de Estructura de la Materia (IEM-CSIC), Consejo Superior de Investigaciones Cientificas, Serrano 121, 28006 Madrid, Spain

E-mail: murai@dipole7.kuic.kyoto-u.ac.jp; j.sanchez@csic.es; j.gomez.rivas@tue.nl

\section{Contents}

1 Extinction for the TM-polarized light: Experiment and calculation

2 Contributions of in- and out-of-plane components to the extinction

3 BICs in the Si nanodisk array without material absorption

4 Effect of thermal annealing on dielectric function of Si 


\section{Extinction for the TM-polarized light: Experiment and calculation}

For TM polarization, where the incident light is polarized along the $x$-axis, the ED in the nanodisks oscillates in the $x$-direction. The scattering field by the dipole moment $p_{x}$ is maximum in the orthogonal direction $\left(y\right.$-direction), coupling preferentially to the $m_{1}=0$, $m_{2}= \pm 1$ diffraction orders as the angle of incidence is changed in the $z-x$ plane (see experimental spectra in Fig.S1.). In contrast, in-plane MD $\left(m_{y}\right)$ couples to the $m_{1}= \pm 1$, $m_{2}=0$ diffraction orders. Following the dispersion of the diffraction, $m_{y}$ is dispersive while $p_{x}$ is non-dispersive. Out-of-plane $\mathrm{ED}, p_{z}$, is excited with the $m_{y}$. Because they spectrally overlap each other, $p_{z}$ cannot be decoupled from $m_{y}$.

The extinction spectra are calculated by CEMD in Fig.S2. It is noted that the complexity of the bands appearing in the wavelength interval $\lambda(\mathrm{nm})=550-650$ fully reproduces the measurements. The lowest-energy features in the range $\lambda(\mathrm{nm})=550-650$ emerge from a combination of the in-plane ED resonance ( $p_{x}$, flat broad band) with two highly-dispersive bands that bend toward the diffraction line at lower frequencies: the in-plane MD resonance $\left(m_{y}\right)$ and the out-of-plane ED resonance $\left(p_{z}\right)$. Interestingly, the latter may give rise also to a $\mathrm{BIC}$ if the aspect ratio of the disks is such that this vertical ED resonance is the lowest-energy one, as exploited in Ref. ${ }^{1}$ through pillar-like meta-atoms. The in-plane MD resonance, on the other hand, gives also rise to the dispersionless broad band about $\lambda=500 \mathrm{~nm}$; higher-order modes arise below $\lambda=550 \mathrm{~nm}$ that are not accounted for in our CEMD calculation. 

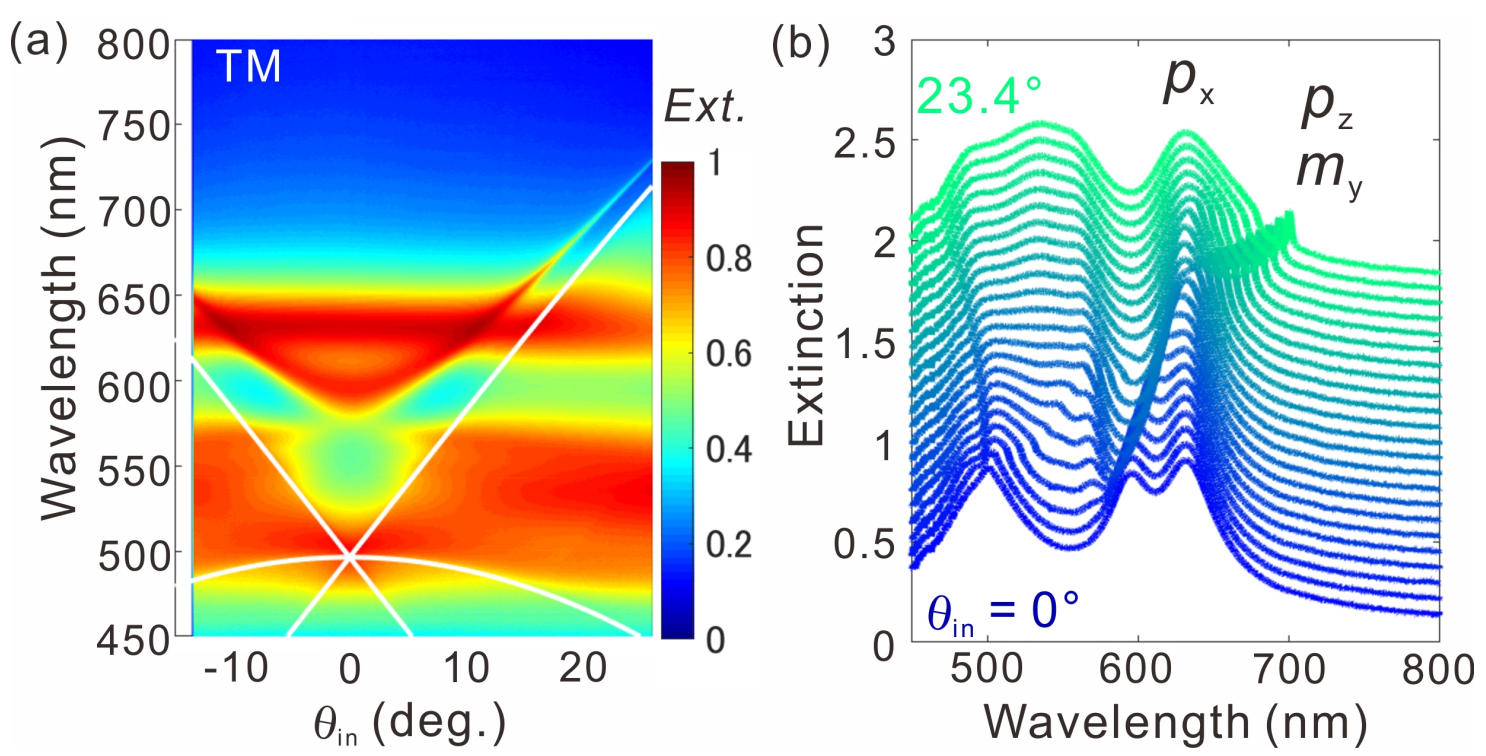

Figure S 1: (a) Experimental extinction of the nanodisk array (period $=340 \mathrm{~nm}$, height $80 \mathrm{~nm}$ and radius $124 \mathrm{~nm}$ ) for TM-polarized light. (b) Cuts of the extinction with varied angle of incidence for TE-polarized light. The spectra are shifted vertically for the sake of clarity.
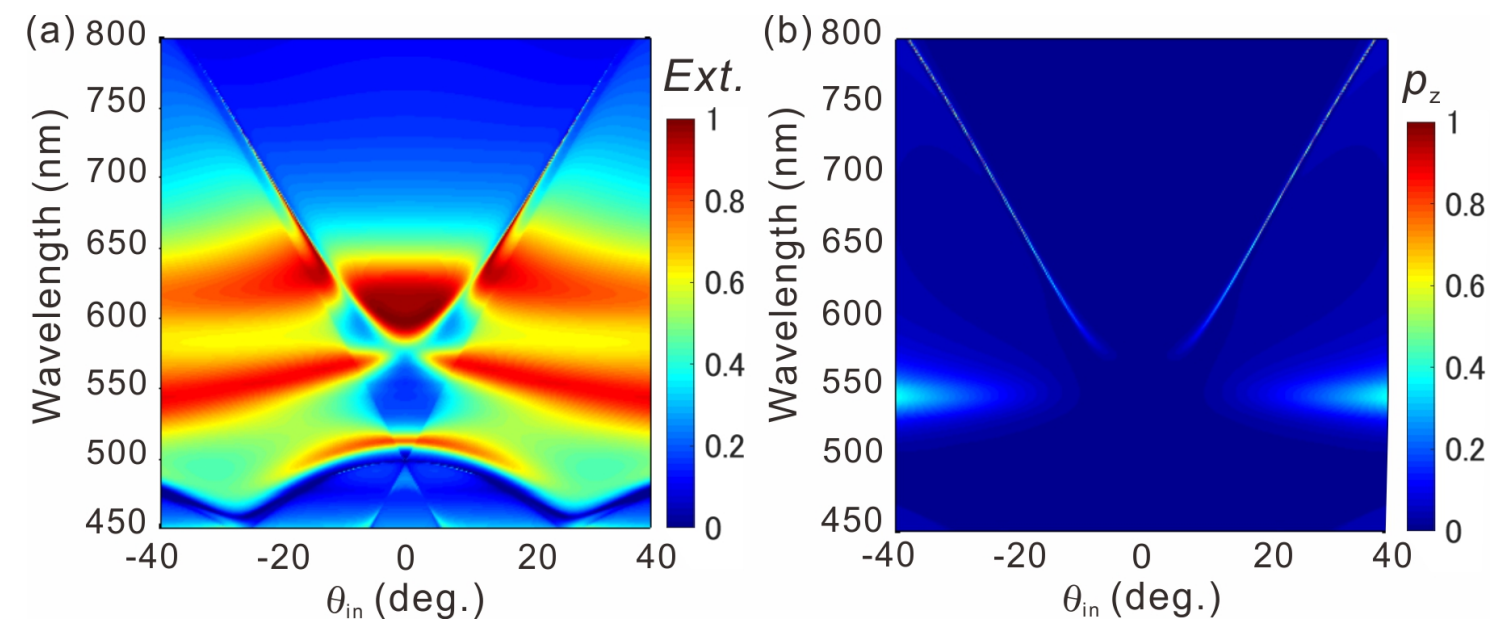

Figure S 2: (a) Calculated (CEMD) extinction of the nanodisk array (period $=340 \mathrm{~nm}$, height 80 $\mathrm{nm}$ and radius $110 \mathrm{~nm}$ ) for TM-polarized light. (b) Contributions from the out-of-plane ED $\left(p_{z}\right)$ component. 


\section{Contributions of in- and out-of-plane components to the extinction}

For a more detailed analysis on the origin of the features, we decompose the extinction spectra into the contributing EDs and MDs. For TE polarization, where the electric field oscillates in the $y$-direction, MD and ED are excited along the $x$ - and $y$-directions, i.e., $m_{x}$ and $p_{y}$, respectively. $m_{x}$ and $p_{y}$ are coupled to the $(0, \pm 1)$ and $(-1,0)$ diffraction orders, respectively. For TM polarization, the $x$ - and $y$-components are flipped. Dispersion of the $z$ components ( $m_{z}$ and $p_{z}$ for TE and TM, respectively) is similar to that of the $y$-component because they are associated to the excitation of the the $y$-component.

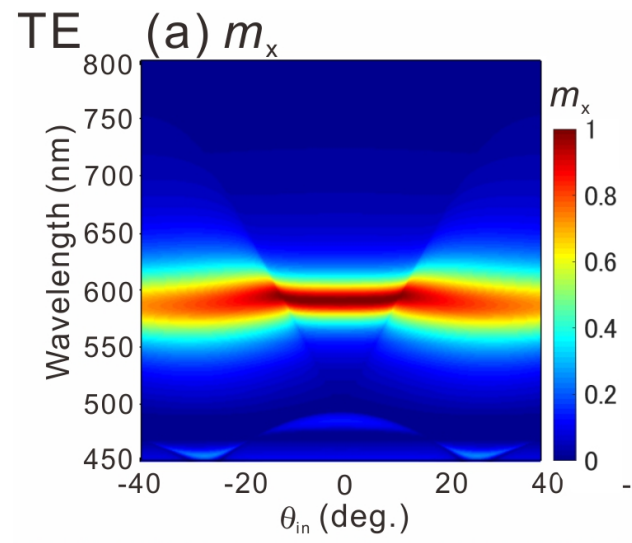

(b) $p_{\mathrm{y}}$

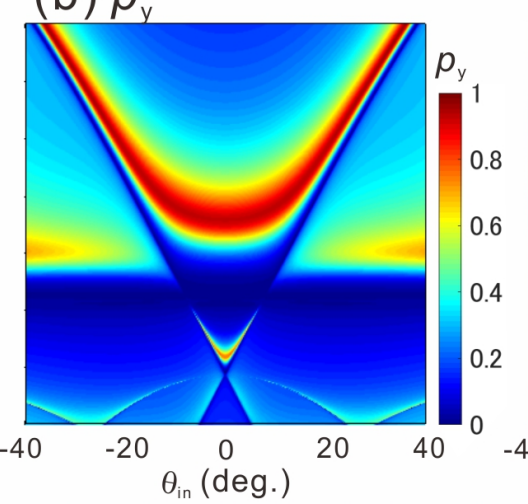

(c) $m_{\mathrm{z}}$
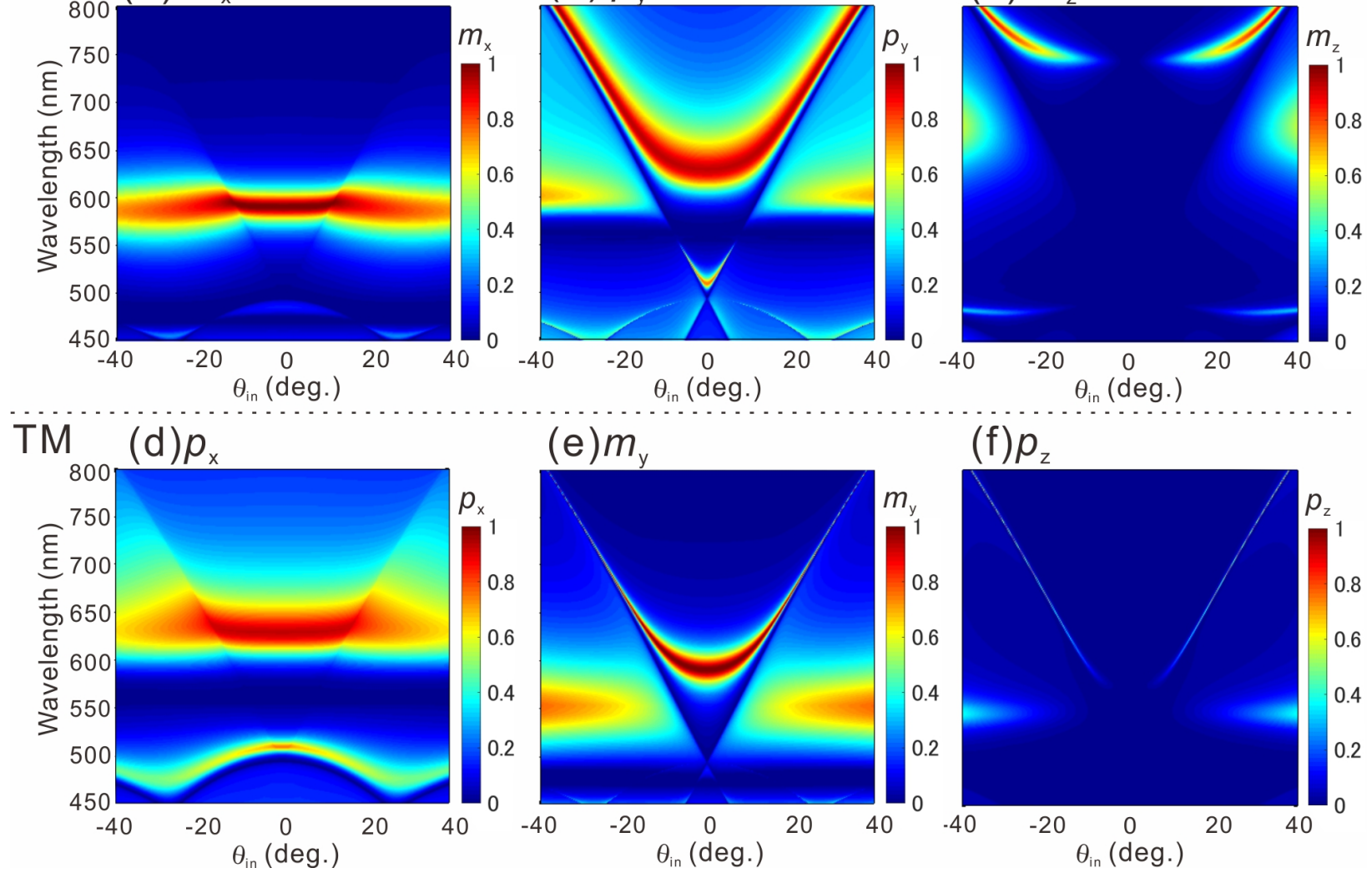

(e) $m_{\mathrm{y}}$

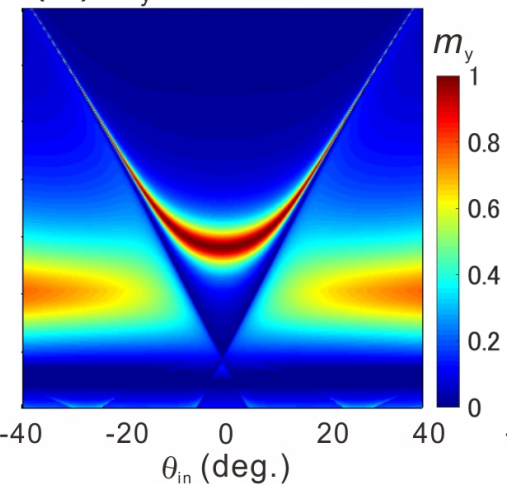

(f) $p_{z}$

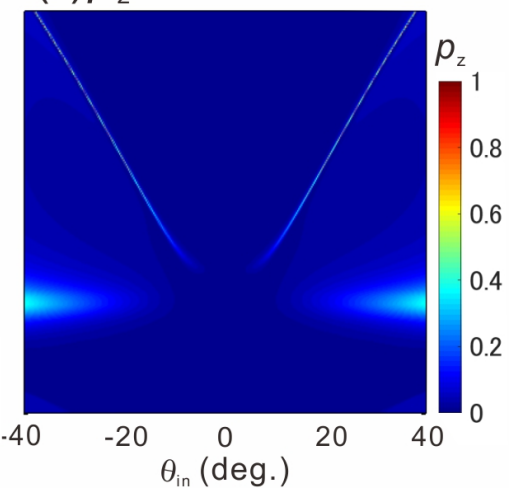

Figure S 3: Analysis of the contributions of electric and magnetic dipoles on the extinction calculated by a multipolar decomposition of the scattering spectra for the nanodisk array (period $=340 \mathrm{~nm}$, height $80 \mathrm{~nm}$ and radius $110 \mathrm{~nm}$ ): (a) $m_{x}$, (b) $p_{y}$, (c) $m_{z}$ for TE-polarized light, and (d) $p_{x}$, (e) $m_{y}$, (f) $p_{z}$ for TM-polarized light. 


\section{BICs in the Si nanodisk array without material absorp- tion}

To analyse the effect of intrinsic absorption of Si on the BIC mode, we calculate the extinction spectra without considering absorption of Si in Fig.S4. Here Si is regarded as a non-absorbing material with dispersionless $n=\sqrt{14}$ and $k=0$, surrounded by a homogeneous medium with $n=\sqrt{2.1}$. As expected, the linewidth of this BIC for TE polarization is much narrower than that with absorption, while the spectral position does not change.
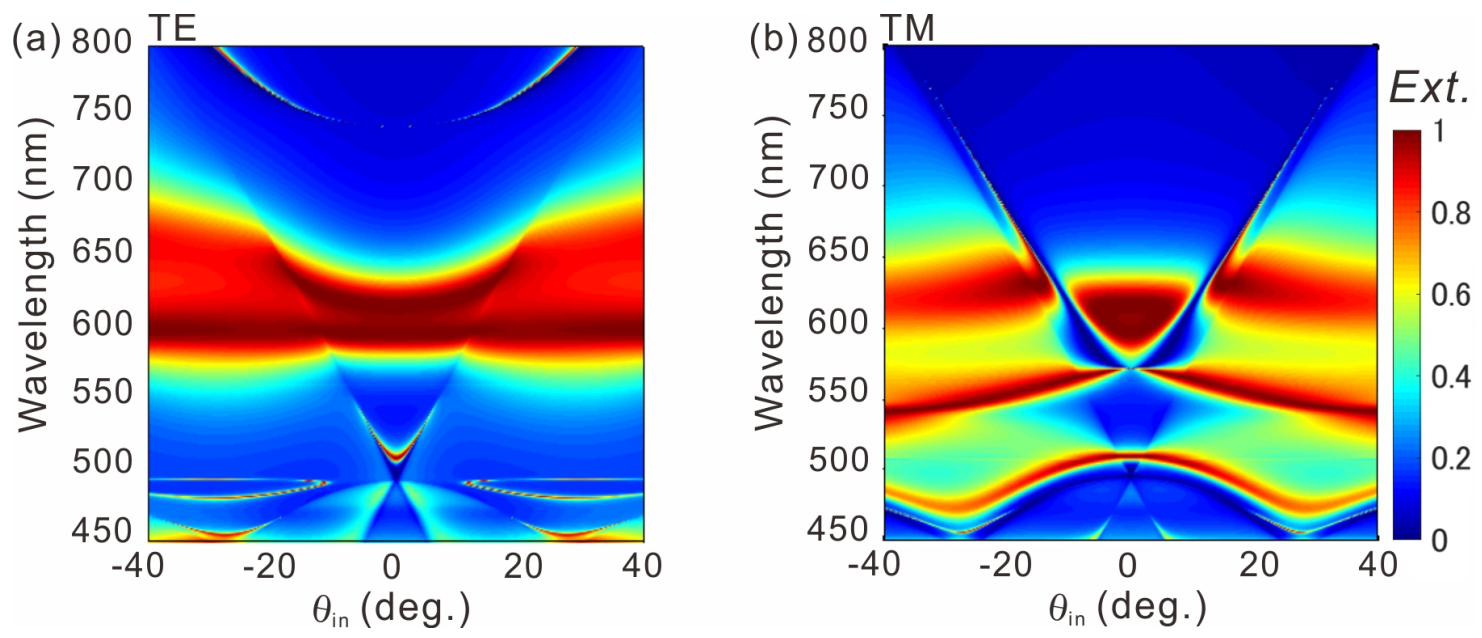

Figure S 4: Calculated extinction of the nanodisk array (period $=340 \mathrm{~nm}$, height $80 \mathrm{~nm}$ and radius $110 \mathrm{~nm}$ ) for (a) TE- and (b) TM polarized light. Dispersionless $n=\sqrt{14}$ and $k=0$ are used for Si. 


\section{Effect of thermal annealing on dielectric function of $\mathrm{Si}$}

We modify the absorption of Si by using rapid thermal annealing (RTA), which enables a rise of temperature with a high rate (typically $\sim 20^{\circ} \mathrm{C} / \mathrm{s}$ ) using halogen lamps. RTA allows local diffusion of atoms while suppressing global deformation of the materials, and is used in Si-based semiconductor fabrication processes. We measure spectroscopic ellipsometry and extract the dielectric function. By increasing the treatment temperature, both real and imaginary parts of the dielectric function decreases as in Fig.S5.

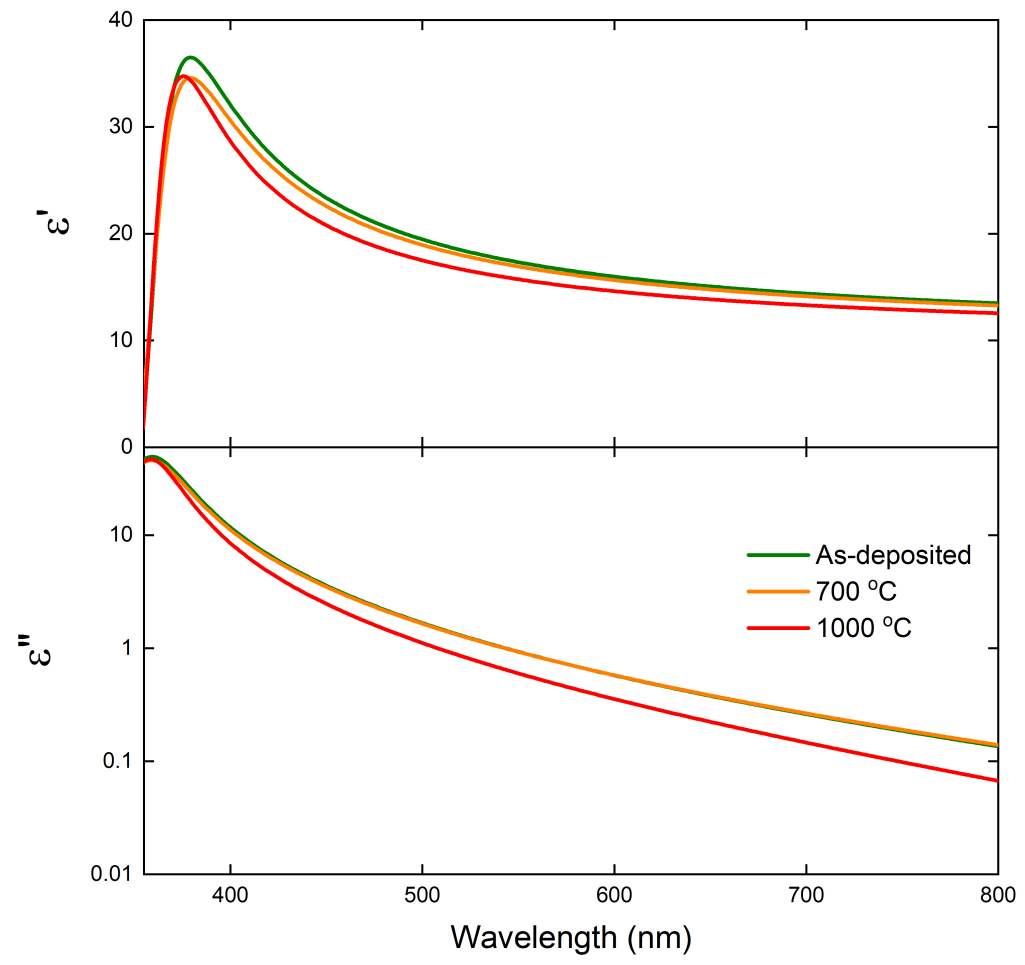

Figure S 5: Real and imaginary parts of the dielectric constants for the Si films as-deposited and after rapid thermal annealing treatment at different temperatures. 


\section{References}

(1) Ha, S. T.; Fu, Y. H.; Emani, N. K.; Pan, Z.; Bakker, R. M.; Paniagua-Domínguez, R.; Kuznetsov, A. I. Directional lasing in resonant semiconductor nanoantenna arrays. Nat. Nanotechnol. 2018, 13, 1042-1047. 